\title{
Investigation of Charge Injection Threshold Field in Epoxy Resin
}

\author{
Miao He*, Dayuan Qiang, Ning Liu, George Chen and Paul L. Lewin \\ The Tony Davies High Voltage Laboratory \\ Electronics and Computer Science Department, \\ University of Southampton, \\ Southampton, UK \\ SO7 1BJ \\ *mh3e12@soton.ac.uk
}

\begin{abstract}
In this paper, space charge formation in epoxy resin has been measured at different applied electric fields using the pulsed electroacoustic technique to reveal the charge injection threshold field. The influence of electrode material on the charge injection has also been investigated. It has been found that the threshold field value for negative charge injection is much lower than that of positive charges, and electrons are the dominant type of charge carrier in epoxy resin. Compared with aluminium, the sample with gold electrode shows a higher threshold field value and lower amount of charge injection. This observation can be attributed to a higher work function of the gold.
\end{abstract}

Keywords - space charge; PEA; threshold; epoxy resin

\section{INTRODUCTION}

Partial discharge (PD) is widely studied under HVAC conditions, including its detection techniques, mechanism and characteristics. However, not much work has been published for PD under HVDC conditions. It is widely anticipated that space charge will influence PD behaviour under DC voltages. Firstly, space charge can distort the local field in the insulation, and may lead to unpredicted insulation failure and tend to influence the DC PD behaviour. Secondly, due to space charges, defects can be introduced into the dielectric in service, and this varies with the field level: if the local field is critically high, new defects with destructive size may appear in a short time [1].

The space charge injection threshold is defined as the electric field level below which space charge injection can be neglected. Recent reported research has considered the charge injection threshold for polyethylene [2-4]. For epoxy resin, however, little work can be found. Epoxy resin is widely applied in the electrical and electronic devices, such as cable joints, protective coatings, and mountings of light emitting diodes because of its high stability to solvents and mechanical shock, as well as excellent dielectric properties. Epoxy resin is also popular in automobile, communication satellites and other aerospace applications, where the requirements for the material quality are strict $[5,6]$.

Unlike polyethylene, epoxy resin is a typical polar material and dipolar polarization takes place when it is subjected to an electric field. Consequently, space charges can be blocked at the interface [7], resulting in a locally high permittivity.
Polarization is more apparent at low frequency and high temperature and it, therefore, increases the difficulty in analysing space charge dynamics in the material [7].

The charge injection threshold value is arguably asymmetric for positive and negative charges. Negative charges are injected from a cathode and move towards an anode, while electrons are extracted from the material at the anode (positive charges are considered to be injected). The aim of the present paper is to determine the charge injection threshold field for epoxy resin and the dominant type of charge carrier. The PEA technique has been applied to study the charge injection situation and space charge dynamics. In addition, the influence of the electrode material on the charge injection threshold field and charge dynamics has also been investigated.

\section{SAMPLE MANUFACTURE}

Fig. 1 shows the mould used for sample manufacture. DER 332, a highly pure bisphenol-A diglycidylether, was selected as the resin and Jeffamine D-230 as the hardener.

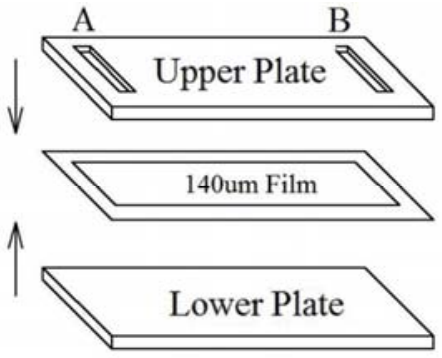

Fig. 1. Mould for sample manufacture.

For space charge measurements with the PEA system used for this research, the sample thickness should be around 140 $\mu \mathrm{m}$. To achieve this thickness with the moulding unit, it was found that $6 \mathrm{~g}$ of resin and hardener are required. The suggested weight ratio of resin to hardener is 1000 to 344 [8]. To avoid cavity formation in the sample, the mixture was degassed for 30 minutes at $50{ }^{\circ} \mathrm{C}$, before it was poured into the mould from the entrance A shown in Fig. 1. The mixture flowed slowly and filled the mould. It was further heated for another 4 hours at $120{ }^{\circ} \mathrm{C}$ to completely cure the resin. Sample thickness depends on the spacer between two plates (in this 
case, $140 \mu \mathrm{m})$. Finally, the sample was naturally cooled to the room temperature.

For the PEA system used in this research, the electrode materials for top and bottom were different, i.e. semiconducting polymer for the top electrode (to achieve better acoustic match) and aluminium for the bottom electrode. To better understand charge injection, some samples were gold-coated on both sides. The thickness of the coated gold electrode is in the order of nano-meters, and it has been proven that such a thin layer does not have significant impact on acoustic impedance [9].

\section{EXPERIMENT PROCESSES}

The Pulsed Electro-Acoustic (PEA) technique is a popular tool to investigate space charge in solid dielectrics. The basic principle has been outlined in [10]. Through the interaction between charge and the applied pulse electric field, acoustic pressure is generated and this pressure propagates through the sample and finally is detected by the piezoelectric transducer attached to the aluminium electrode (Fig. 2.). The electric signal detected by the transducer contains the detailed information of space charge in terms of location and magnitude. The quantitative information can be extracted via a process of calibration/deconvolution.

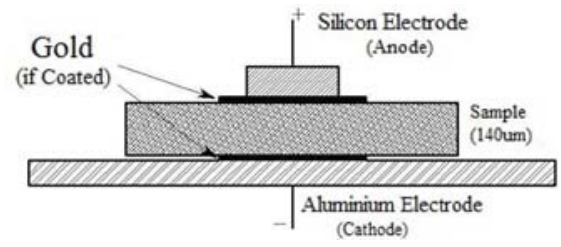

Fig. 2. Cross section of the PEA electrode and sample system.

To determine the charge injection threshold field, the applied field level increased from $5 \mathrm{kV} \cdot \mathrm{mm}^{-1}$ to $10 \mathrm{kV} \cdot \mathrm{mm}^{-1}$ with the step of $1 \mathrm{kV} \cdot \mathrm{mm}^{-1}$. To investigate the influence of injected charge on the electric field in the sample, a step of $10 \mathrm{kV} . \mathrm{mm}^{-1}$ was used from $10 \mathrm{kV} \cdot \mathrm{mm}^{-1}$ to $50 \mathrm{kV} \cdot \mathrm{mm}^{-1}$. The lowest applied voltage level was selected to be $500 \mathrm{~V}$ $\left(3.57 \mathrm{kV} . \mathrm{mm}^{-1}\right.$ for $140 \mu \mathrm{m}$ sample) and the measured voltage signal was used as the reference signal (calibration). The applied voltage was adjusted according to the small variation in sample thickness to ensure that the applied electric field as intended.

The data recording procedure was the same for all field levels, i.e. data was acquired every 30 seconds in the first 5 minutes and then every 5 minutes until it 30 minutes had elapsed. The tests were repeated at least two times for each field level to ensure results obtained were repeatable and reliable.

Recorded data was analysed using the subtracted method, where the effect of both the applied voltage and pulse voltage can be removed. The resulting charge profile is regarded as the injected amount of charge, including fast (mobile) charges, i.e.

$$
\rho_{a c c}(x)=\rho_{a p p}(x)-\frac{V_{a p p}}{V_{r e f}} \rho_{r e f}(x) .
$$

where $\rho_{a c c}(x)$ is the subtracted charge density, and $\rho_{a p p}(x)$ and $\rho_{\text {ref }}(x)$ are charge density at applied and reference voltage respectively. $V_{a p p}$ and $V_{\text {ref }}$ are applied and reference voltage [11].

\section{RESULTS AND DISCUSSION}

In this part, the results for the uncoated and gold-coated samples are given, followed with analysis and explanation.

\section{A. Experiment on Uncoated Samples}

Fig. 3 shows the charge profile at $500 \mathrm{~V}$ over 1800 s and the reference signal was recorded at $15 \mathrm{~s}$. It is seen that even at $500 \mathrm{~V}\left(3.57 \mathrm{kV} . \mathrm{mm}^{-1}\right)$, electrons, are injected from the cathode and move towards the anode. Comparably, no positive charge injection is seen. The injection threshold is not symmetric for positive and negative charges. Firstly, the mechanism of injection differs in positive and negative charges as mentioned earlier. Secondly, the asymmetry can also be explained by the difference in electrode material. The cathode is the aluminium electrode, and the anode is a semi-conductive electrode. The charge injection threshold field observed here is much lower than $10 \mathrm{kV} \cdot \mathrm{mm}^{-1}$, a value commonly recognised $[2,4]$ for many polymers. We believe that the subtraction method used here includes the contribution from mobile charges which are excluded when using the "volts-off" measurement technique in quantifying space charge. It is more sensitive and able to detect the charge injection threshold field more accurately.

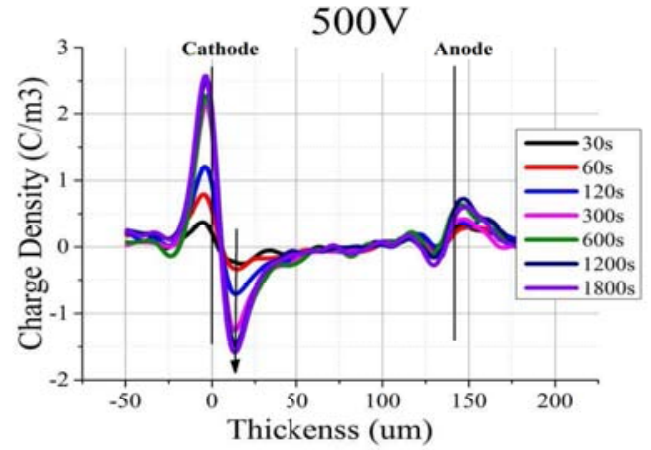

Fig. 3. Charge profile at $500 \mathrm{~V}$ under subtraction method in uncoated samples in 1800 s.

Subtracted charge profiles at various field levels are illustrated in Fig. 4. It can be seen that the charge injection threshold field for electron injection is low, while the value for positive charge injection is as high as $30 \mathrm{kV} \cdot \mathrm{mm}^{-1}$. Besides, the transportation velocity of positive charges is much slower than that of negative charges. Therefore, electrons play a dominant role in space charge activity at low fields. Even at a field where positive charges appear, electrons are also important because of their fast movement. At low fields (5 $\mathrm{kV} \cdot \mathrm{mm}^{-1}$ ), the fluctuation can be seen in the obtained data due to background noise and the limited sensitivity of the PEA system. 


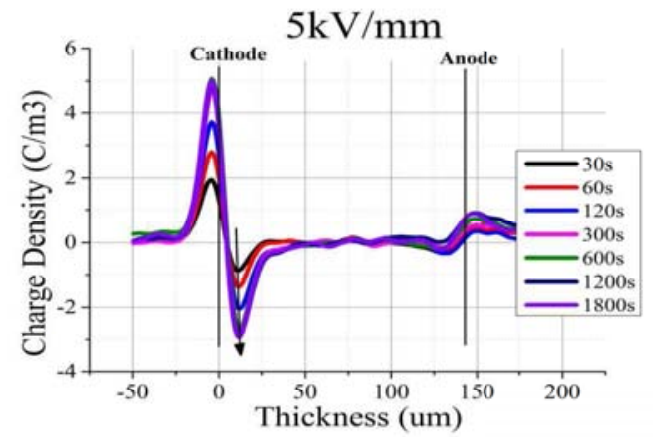

(a)

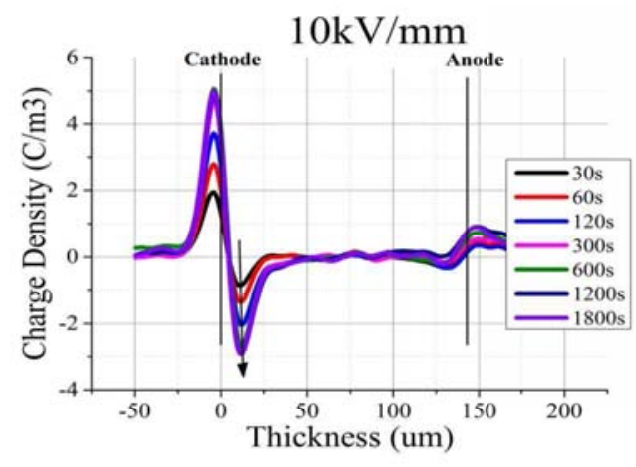

(b)

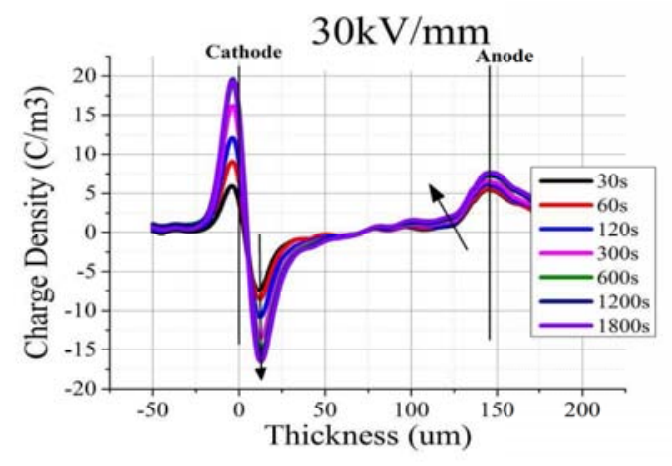

(c)

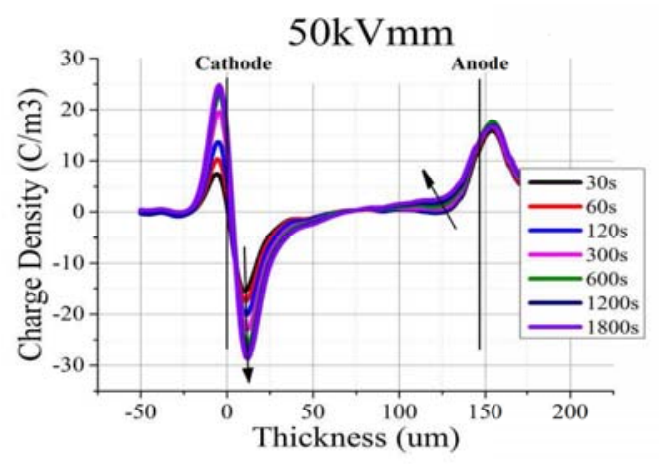

(d)

Fig. 4. Charge profile at a) $5 \mathrm{kV} \cdot \mathrm{mm}^{-1}$; b) $10 \mathrm{kV} \cdot \mathrm{mm}^{-1}$; c) $30 \mathrm{kV} \cdot \mathrm{mm}^{-1}$ and d) $50 \mathrm{kV} \cdot \mathrm{mm}^{-1}$ in uncoated sample under subtraction method in $1800 \mathrm{~s}$.
The injected electron and positive charge for all the applied fields is summarised in Fig. 5 and Fig. 6 respectively. It can be seen that the charge injection rate is higher during the early stage of voltage application. The intensity of charge injection is enhanced by the applied electric field. The amount of electrons are much higher than that of positive charge. For example, at $50 \mathrm{kV} \cdot \mathrm{mm}^{-1}$, nearly $36 \mathrm{nC}$ of electron charge is injected into the resin after 30 minutes, while only $15 \mathrm{nC}$ of positive charge has been injected. The fluctuation in positive charge accumulation shown in Fig. 6 may reflect the fact that there is recombination with electrons. Under the influence of the electric field, electrons may move from the injected region towards the anode, resulting in recombination with positive charge. The time period, 1800s, is believed to be long enough for determining the charge injection threshold value and it also allows one to observe the effect of voltage duration on the charge injection.

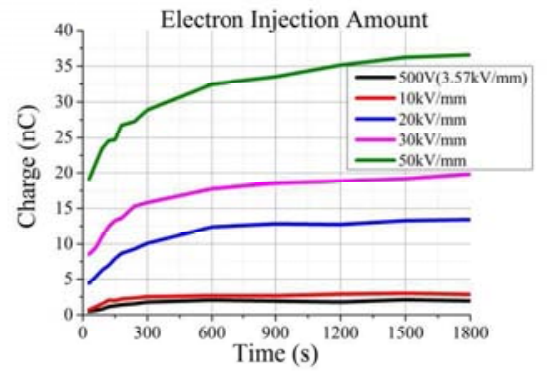

Fig. 5. Electron injected amount in uncoated samples in 1800s.

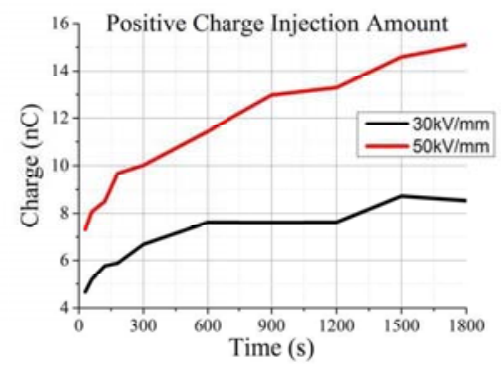

Fig. 6. Positive charge injection amount in uncoated samples in 1800s.

\section{B. Experiment on Gold-coated Samples}

The work function value indicates the ease of electron escaping from a material. Higher work function means that electrons are difficult to escape from the outermost molecular layer and enter the material. To understand the influence of electrode on charge injection, space charge measurements were carried out on the gold-coated samples. The work function of aluminium is $4.08 \mathrm{eV}$, while that of gold is $4.70 \mathrm{eV}$ [12]. Therefore, it is deduced that the charge injection amount should be reduced for samples with gold-coated electrodes.

Fig. 7 shows the subtracted charge profiles, and Fig. 8 illustrates the electron injection amount obtained from the gold-coated samples. Charge injection is greatly reduced when the gold electrode was used. For example, $15 \mathrm{nC}$ electron can be injected into the epoxy resin under $30 \mathrm{kV} \cdot \mathrm{mm}^{-1}$ after $300 \mathrm{~s}$ with an aluminium electrode, which is much higher than the situation for gold electrode after $1800 \mathrm{~s}$ at $50 \mathrm{kV} \cdot \mathrm{mm}^{-1}$. The 
observed phenomenon is consistent with the difference in the work function for two electrode materials. The injection rate is similar to that of the aluminium electrode case, i.e. the injection rate is fast at the beginning and decreases with time.

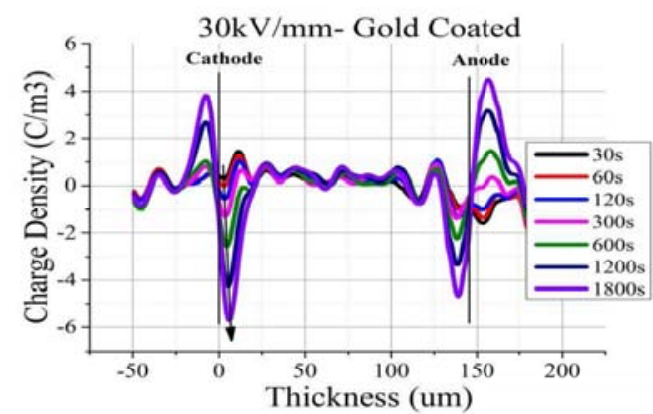

(a)

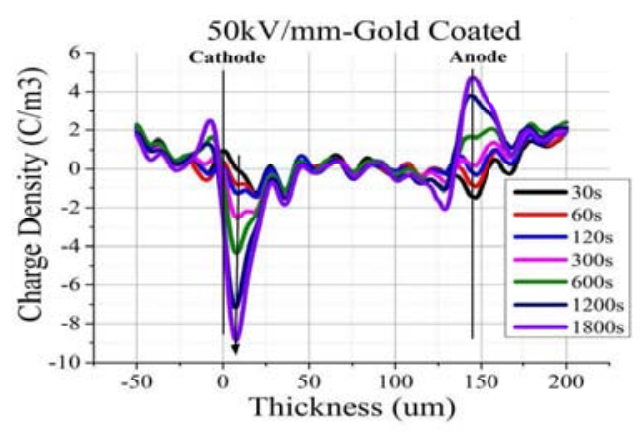

(b)

Fig. 7. Charge profile at a) $30 \mathrm{kV} \cdot \mathrm{mm}^{-1}$; b) $50 \mathrm{kV} \cdot \mathrm{mm}^{-1}$ in gold-coated samples under subtraction method in $1800 \mathrm{~s}$.

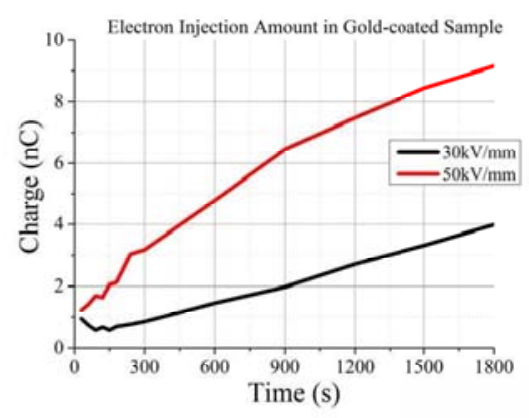

Fig. 8. Electron injected amount in gold-coated samples in 1800 s.

\section{CONCLUSIONS}

In this paper, the charge injection threshold for space charge for an epoxy resin has been investigated using the PEA technique. The threshold for charge injection is not symmetric because of different injection mechanisms for positive and negative charges and the use of asymmetric electrode materials. Electrons should always be considered when epoxy resin is under high voltages as the injection threshold electric field is lower than $3.57 \mathrm{kV} . \mathrm{mm}^{-1}$. The threshold for positive charge injection can be as high as $30 \mathrm{kV} . \mathrm{mm}^{-1}$. Furthermore, negative charges are transported faster than positive charges, and more electrons are injected into the material than positive charges.
Therefore, electrons are the dominant charge carriers in epoxy resin.

The work function of the electrode material has a significant impact on the charge injection. Compared with an aluminium electrode, charge injection amount is dramatically reduced when a gold electrode is used. Charge injection is faster in the first stage and the rate of injection decreases with time.

The injected charge carriers will affect the electrical performance of epoxy resin including PD characteristics under HVDC conditions. This will be reported in future work.

\section{REFERENCES}

[1] Y. Shibuya, S. Zoledziowski, and J. H. Calderwood, "Void formation and electrical breakdown in epoxy resin", IEEE Transactions on Power Apparatus and Systems, Vol. 96, pp. 198207, 1977.

[2] Mary, D.; Laurent, C.; Teyssedre, G.; Bamji, S.; Bulinski, A.; Dakka, M.A.; Cisse, L., "Threshold of space charge injection and electroluminescence in polymeric insulation," IEEE CEIDP pp.249,252, 17-20 Oct. 2004.

[3] An, Zhenlian; Cang, Jun; Chen, Xuan; Liu, Yaqiang, "Method for investigating threshold field of charge injection at electrode/dielectric interfaces by space charge observation," Applied Physics Letters, Vol.101, No.17, pp.172902, Oct 2012.

[4] Montanari, G.C.; Palmieri, F.; Mazzanti, G.; Laurent, C.; Teyssedre, G., "AC charge injection investigated by means of space charge measurements: threshold and frequency dependence," IEEE ICPADM, pp.895-899, 1-5 June 2003.

[5] I. Hamerton, Recent Developments in Epoxy Resins Vol. 8. Shrewsbury, Shropshire, United Kingdom: RAPRA Technology Ltd, 1997.

[6] C. A. May, Epoxy Resins: Chemistry and Technology (Second Edition, Revised and Expanded). New York, United States of America: Marcel Dekker, Inc., 1988.

[7] Tian, F.; Ohki, Y., "Charge transport characteristics in epoxy resin at high temperatures based on electrode polarization analysis," IEEE CEIDP, pp.1318-1321, 20-23 Oct. 2013.

[8] M. Reading, Z. Xu, A. S. Vaughan, and P. L. Lewin, "The thermal and electrical properties of nano-silicon dioxide filled epoxy systems for use in high voltage insulation," IEEE EIC, pp. 493-497, 2011.

[9] Peng Liu; Zhen Xiang; Xin Ning; Hua Feng; Zongren Peng, "Effect of electrode material on space charge behavior in epoxy resin," IEEE CEIDP, pp.230-233, 20-23 Oct. 2013.

[10] Li, Ying; Yasuda, M.; Takada, Tatsuo, "Pulsed electroacoustic method for measurement of charge accumulation in solid dielectrics," IEEE Transactions on Dielectrics and Electrical Insulation, Vol.1, No.2, pp.188-195, 1994.

[11] N. Liu; C. Zhou; and G. Chen, "Different approach to investigate the threshold field of injected charges accumulation," submitted to APL.

[12] O. Gallot-Lavallée, G. Teyssèdre, C. Laurent, and S. Rowe, "Space charge behaviour in an epoxy resin: the influence of fillers, temperature and electrode material," Journal of Physics D: Applied Physics, Vol. 38, pp. 2017-2025, 2005. 